\title{
Alternative translational initiation of ATP sulfurylase underlying dual localization of sulfate assimilation pathways in plastids and cytosol in Arabidopsis thaliana
}

\author{
Anne-Sophie Bohrer ${ }^{1+}$, Naoko Yoshimoto ${ }^{2 t}$, Ai Sekiguchi ${ }^{2}$, Nicholas Rykulski ${ }^{1}$, Kazuki Saito ${ }^{2,3}$ and \\ Hideki Takahashi ${ }^{1 *}$
}

${ }^{1}$ Department of Biochemistry and Molecular Biology, Michigan State University, East Lansing, MI, USA

${ }^{2}$ Graduate School of Pharmaceutical Sciences, Chiba University, Chiba, Japan

${ }^{3}$ RIKEN Center for Sustainable Resource Science, Yokohama, Japan

\author{
Edited by: \\ Ruediger Hell, University of \\ Heidelberg, Germany \\ Reviewed by: \\ Thomas Leustek, Rutgers, The State \\ University of New Jersey, USA \\ Andreas Wachter, University \\ Tuebingen, Germany \\ *Correspondence: \\ Hideki Takahashi, Department of \\ Biochemistry and Molecular Biology, \\ Michigan State University, 603 \\ Wilson Road, 209 Biochemistry \\ Building, East Lansing, MI 48824, \\ USA \\ e-mail:htakaha@msu.edu \\ ${ }^{t}$ These authors have contributed \\ equally to this work.
}

Plants assimilate inorganic sulfate into sulfur-containing vital metabolites. ATP sulfurylase (ATPS) is the enzyme catalyzing the key entry step of the sulfate assimilation pathway in both plastids and cytosol in plants. Arabidopsis thaliana has four ATPS genes (ATPS1, $-2,-3$, and -4) encoding ATPS pre-proteins containing N-terminal transit peptide sequences for plastid targeting, however, the genetic identity of the cytosolic ATPS has remained unverified. Here we show that Arabidopsis ATPS2 dually encodes plastidic and cytosolic ATPS isoforms, differentiating their subcellular localizations by initiating translation at AUGMet1 to produce plastid-targeted ATPS2 pre-proteins or at AUGMet52 or AUGMet58 within the transit peptide to have ATPS2 stay in cytosol. Translational initiation of ATPS2 at AUGMet52 or AUGMet58 was verified by expressing a tandem-fused

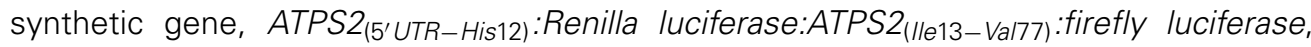
under a single constitutively active CaMV $35 \mathrm{~S}$ promoter in Arabidopsis protoplasts and examining the activities of two different luciferases translated in-frame with split $\mathrm{N}$-terminal portions of ATPS2. Introducing missense mutations at AUGMet52 and AUGMet58 significantly reduced the firefly luciferase activity, while AUGMet52 was a relatively preferred site for the alternative translational initiation. The activity of luciferase fusion protein starting at $A \cup G^{M e t 52}$ or $A \cup G M e t 58$ was not modulated by changes in sulfate conditions. The dual localizations of ATPS2 in plastids and cytosol were further evidenced by expression of ATPS2-GFP fusion proteins in Arabidopsis protoplasts and transgenic lines, while they were also under control of tissue-specific ATPS2 promoter activity found predominantly in leaf epidermal cells, guard cells, vascular tissues and roots.

Keywords: sulfur metabolism, ATP sulfurylase, alternative translational initiation, dual localization, Arabidopsis

\section{INTRODUCTION}

Sulfur is an essential macronutrient for plant growth, and it can be found in a wide variety of cellular components such as Cys, Met, GSH, sulfolipids, redox centers, and specialized metabolites involved in biotic and abiotic responses (Halkier and Gershenzon, 2006; Shimojima, 2011; Takahashi et al., 2011; Noctor et al., 2012). Sulfate is the main sulfur source available for plants in the environment. Therefore, reducing cofactors and carbon skeletons generated through photosynthesis are required in order to assimilate sulfate into organic sulfur metabolites. Following uptake of sulfate across the plasma membranes, several metabolic steps serve for reduction of sulfate. The first step of the sulfate assimilation pathway is catalyzed by ATP sulfurylase (ATPS) (ATP: sulfate adenylyltransferase, EC: 2.7.7.4) which uses ATP and sulfate to yield adenosine $5^{\prime}$-phosphosulfate (APS) and pyrophosphate. Following this step, APS is subsequently phosphorylated by APS kinase (APK, EC: 2.7.1.25) to form $3^{\prime}$-phosphoadenosine $5^{\prime}$ phosphosulfate (PAPS). PAPS is then used as a donor for sulfation reactions. Besides being utilized in the phosphorylation pathway, APS is supplied to the reductive assimilation pathway where it is converted to sulfite by APS reductase (APR, EC: 1.8.99.2). Sulfite is then reduced to sulfide by sulfite reductase ( $\mathrm{SiR}, \mathrm{EC}$ : 1.8.7.1). Cys biosynthesis from sulfide and $O$-acetylserine occurs following this reductive assimilation pathway.

ATPS is encoded by a multigenic family and its activity can be detected in cytosol and chloroplasts in plants (Lunn et al., 1990; Renosto et al., 1993; Klonus et al., 1994; Leustek et al., 1994; Murillo and Leustek, 1995; Logan et al., 1996; Hatzfeld et al., 2000; Rotte and Leustek, 2000; Phartiyal et al., 2006). APK is also found in both cytosol and plastids (Lee and Leustek, 1998; Lillig et al., 2001; Mugford et al., 2009). In contrast, reduction of APS catalyzed by APR occurs only in the plastids (Gutierrez-Marcos et al., 1996; Setya et al., 1996; Rotte and Leustek, 2000; Suter et al., 2000). APK and APR therefore compete for their common substrate APS in plastids, while no such competition happens in cytosol. PAPS biosynthesis in cytosol appears simple with ATPS 
and APK being direct enzymes involved in the pathway. However, it can eventually be affected by metabolic fluxes of APS phosphorylation and reduction in plastids, because PAPS is transported from plastids to cytosol (Gigolashvili et al., 2012). Thus, ATPS activities in plastids and cytosol contribute to provision of APS for downstream pathways in different ways, and their roles may vary depending on subcellular localizations.

Four ATPS genes (ATPS1, $-2,-3$, and -4 ) are present in the Arabidopsis genome (Leustek et al., 1994; Klonus et al., 1995; Murillo and Leustek, 1995; Logan et al., 1996; Hatzfeld et al., 2000). The protein coding regions of all four ATPS have the N-terminal leader sequences with characteristics for plastidtargeting transit peptides followed by the ATPS catalytic domains. Despite the presence of transit peptides in all four ATPS, the ATPS activity is detected in both chloroplasts and cytosol in Arabidopsis leaves (Rotte and Leustek, 2000). Thus, the identity of cytosolic ATPS has remained arguable, although ATPS2 (Logan et al., 1996) has been proposed as a candidate gene to encode two isoforms (i.e., plastid- and cytosol-localizing ATPS) based on prediction of alternative translational initiation sites within the $\mathrm{N}$-terminal transit peptide region (Hatzfeld et al., 2000).

In this study, we demonstrate experimental evidence that ATPS2 is alternatively translated into two different isoforms that dually localize in plastids and cytosol in Arabidopsis. The present study provides new insights into molecular mechanisms differentiating sulfate assimilation pathways in plastids and cytosol in plants.

\section{MATERIALS AND METHODS CHIMERIC GENE CONSTRUCTS FOR PROTOPLAST TRANSFECTION}

Chimeric genes were generated using overlap-extension PCR methods. All the independent gene fragments were first amplified by PCRs using overlapping primers (Supplemental Table S1). The full-length chimeric genes were subsequently amplified by PCRs using $50 \mathrm{ng}$ of each independent gene fragment, obtained from the initial PCRs, as templates and the primer pairs 1F/4R for ATPS2-dual-Luc, 1F/6R for ATPS2 ${ }_{\left(5^{\prime} U T R-V a l 77\right)^{-}}$ GFP and $A T P S 2_{F L}-G F P$, and 9F/6R for $A T P S 1_{\left(5^{\prime} U T R-V a l 63\right)}-G F P$ genes (Supplemental Table S1). All PCRs were performed using Platinum Pfx DNA Polymerase (Thermo Fisher Scientific). The resultant PCR-amplified chimeric genes were cloned into pCRBlunt II-TOPO vector (Thermo Fisher Scientific) and fully sequenced. Each BamHI-NotI-ended chimeric gene was ligated with the BamHI-NotI fragment of p35s:GFP vector using a ligation kit Ligation Mighty Mix (Takara Bio) to generate $p 35 S$ :ATPS2-dual-Luc, p35S:ATPS2 ${ }_{\left(5^{\prime} U T R-V a l 77\right)}-G F P$, p35S:ATPS $2_{F L}-G F P$, and p35S:ATPS1 ${ }_{\left(5^{\prime} U T R-V a l 63\right)}-G F P$.

The p35S:GFP vector [CaMV 35S:sGFP(S65T)] used in this study is a modified version of the $35 \Omega-s G F P(S 65 T)$ vector (Chiu et al., 1996) from which the $35 \Omega$ promoter sequence was removed and replaced by the CaMV 35S promoter sequence of pBI221 (Clontech). The HindIII-BamHI fragment (vector backbone, $3.65 \mathrm{~kb})$ of $35 \Omega-s G F P(S 65 T)$ and the HindIII-BamHI fragment (CaMV 35S promoter, $0.8 \mathrm{~kb}$ ) of pBI221 were ligated to obtain p35S:GFP.

Mutated versions of p35S:ATPS2-dual-Luc and p35S: ATPS2 $\left(5^{\prime} U T R-\right.$ Val77)-GFP were generated by site-directed mutagenesis using the QuickChange Lightning Site-Directed Mutagenesis Kit (Agilent) according to the manufacturer's instructions. Oligonucleotide primers and DNA templates that were used to introduce various point mutations are listed in Supplemental Tables S2, S3.

\section{PROTOPLAST ISOLATION AND TRANSFECTION}

The protoplast isolation and transfections were performed as described by Yoo et al. (2007). Arabidopsis wild-type (Col-0) plants were grown on soil in an environment-controlled chamber under a 12 -h-light / 12 -h-dark cycle at $22^{\circ} \mathrm{C}$ with a light intensity of $80 \mu \mathrm{E} \mathrm{m}^{-2} \mathrm{~s}^{-1}$ and a relative humidity of $50 \%$. For each protoplast transfection, 15-20 $\mu \mathrm{g}$ of plasmid DNAs were used, and the protoplasts were incubated in the transfection mixture for $10 \mathrm{~min}$. Protoplasts were then re-suspended in WI solution (Yoo et al., 2007) supplemented with $1 \mathrm{mM} \mathrm{MgSO}_{4}$ ( $+\mathrm{S}$ condition) or $1 \mathrm{mM} \mathrm{MgCl} 2$ ( $-\mathrm{S}$ condition), and incubated in the dark for $16 \mathrm{~h}$. Protoplasts were then harvested by centrifugation.

\section{DUAL-LUCIFERASE ASSAYS}

Dual luciferase assays were performed with the dual luciferase reporter assay system using the firefly luciferase reagent (LARII) and the Renilla luciferase reagent with firefly quenching (Stop \& Glo) (Promega). All reagents were prepared as described by the manufacturer. Protoplasts were re-suspended in $50 \mu \mathrm{l} 1 \mathrm{X}$ passive lysis buffer and incubated on ice for $15 \mathrm{~min}$. The lysates were then centrifuged for $15 \mathrm{~min}$ at maximum speed at $4^{\circ} \mathrm{C}$. Ten $\mu \mathrm{l}$ of undiluted supernatants were used to monitor the bioluminescence using a Centro SX3 luminometer (Berthold Technologies). Statistical significance was examined by One-Way analysis of the variance (ANOVA) and the Tukey's HSD post-hoc test with the level of significance set at $5 \%$.

\section{CREATION OF TRANSGENIC PLANTS EXPRESSING ATPS2 FUSED WITH GFP}

For the creation of ATPS2pro:ATPS2-GFP fusion gene construct, oligonucleotide primers ATPS2-prom-FSal and ATPS2CDSnstop-RNco (Supplemental Table S4) were used to amplify a genomic DNA fragment of ATPS2 gene starting from $5^{\prime}$-region 3009-bp upstream of the plausible first translational initiation site and terminating just before the translational stop site. PCR was performed on genomic DNA prepared from Arabidopsis thaliana ecotype Col-0 using KOD plus DNA polymerase (Toyobo, Japan). The resultant PCR-amplified fragment of ATPS2 was cloned into pCRBlunt II-TOPO (Thermo Fisher Scientific) and fully sequenced. The SalI-NcoI-ended ATPS2 gene fragment was inserted in the place of $35 \Omega$ in the $35 \Omega$-sGFP(S65T) vector (Chiu et al., 1996) to obtain the ATPS2pro:ATPS2-sGFP(S65T):NOSter fusion gene. This fusion gene fragment was placed between the SalI and EcoRI sites in the binary plasmid, pBI101 (Clontech), replacing the $\beta$-glucuronidase gene and the NOSter region (Figure S1).

The ATPS2pro:ATPS2-GFP chimeric gene constructs with mutated versions of ATPS2 were created as follows. The BamHI and $\mathrm{XbaI}$ sites, respectively located 748-bp upstream and 1127bp downstream of the first translational initiation site of ATPS2, were used to cut out a DNA fragment from the binary plasmid 
harboring the ATPS2pro:ATPS2-sGFP(S65T):NOSter fusion gene (Figure S1), and this BamHI-XbaI fragment was used as a template for overlap-extension PCRs. The nucleotide sequences of primer pairs used for the first PCRs are shown in Supplemental Table S4. The fragments obtained from the first PCR were mixed and amplified by PCR using primers ATPS2(-753)-FBam and ATPS2 $(+1136)$-RXba. The DNA templates and the pairs of primers used for the construction of the mutated versions of ATPS2pro:ATPS2-sGFP(S65T):NOSter fusion gene are detailed in the Supplemental Table S5. The resultant PCR fragments containing mutations in ATPS2 were cloned into pCR-Blunt II-TOPO and fully sequenced. The mutated ATPS2 fragments were cut out as $B a m \mathrm{HI}-\mathrm{XbaI}$ fragments, and used to replace the corresponding region of wild-type ATPS2 gene in the binary plasmid harboring the ATPS2pro:ATPS2-sGFP(S65T):NOSter fusion gene construct (Figure S1).

The binary plasmids were transferred to Agrobacterium tumefaciens C58C1 GV3101 (pMP90) (Koncz and Schell, 1986) by a freeze-thaw method (Höfgen and Willmitzer, 1988). Arabidopsis thaliana ecotype Col-0 plants were transformed by a floral dip method (Clough and Bent, 1998). Transgenic plants were selected on GM agar medium (Valvekens et al., 1988) containing $50 \mathrm{mg}$ $\mathrm{L}^{-1}$ kanamycin sulfate. Kanamycin-resistant $\mathrm{T}_{2}$ progenies were used for the analyses.

\section{MICROSCOPY AND IMAGING OF GFP}

Fluorescence of ATPS2-GFP fusion proteins in protoplasts and transgenic plants was observed using confocal laserscanning microscopes, Fluoview FV10i (Olympus) and LSM510 (Zeiss).

\section{PROTEIN EXTRACTION AND IMMUNOBLOT ANALYSIS}

Total protein was prepared from leaves and roots of plants grown for 2 weeks on GM agar medium (Valvekens et al., 1988). Tissues were ground under liquid nitrogen and homogenized in the extraction buffer [50 mM Tris-MES ( $\mathrm{pH} 7.5$ ), $300 \mathrm{mM}$ sucrose, $150 \mathrm{mM} \mathrm{NaCl}, 10 \mathrm{mM} \mathrm{CH}_{3} \mathrm{COOK}, 5 \mathrm{mM}$ EDTA, $20 \mu \mathrm{M}$ leupeptine, $100 \mu \mathrm{M}$ 4-(2-aminoethyl)benzenesulfonyl fluoride, $1 \mathrm{mM}$ phenylmethylsulfonyl fluoride]. The lysate was centrifuged at $10,000 \mathrm{~g}$ for $15 \mathrm{~min}$, and the supernatant was collected. Protein concentrations were determined using a Bio-Rad protein assaying kit (Bio-Rad) based on the Bradford method (Bradford, 1976), using bovine serum albumin as a standard. Proteins were separated in $10 \%(\mathrm{w} / \mathrm{v})$ polyacrylamide gel, and transferred to Immobilon-P membrane (Millipore) by electroblotting. Ten micrograms of crude proteins were loaded to each lane of the gels. The blot was incubated with anti-GFP mouse monoclonal antibody (Nacalai Tesque, Japan), followed by incubation with goat anti-mouse IgG conjugated to alkaline phosphatase (Promega). The presence of immuno-reactive protein was detected through the use of 5-bromo-4-chloro-3-indolyl-phosphate and nitro blue tetrazolium (Promega).

\section{ACCESSION NUMBERS}

The reference sequence information on Arabidopsis ATPS gene family members is available at The Arabidopsis Information Resource (TAIR, http://www.arabidopsis.org/) under the following accession numbers: ATPS1 (At3g22890); ATPS2 (At1g19920); ATPS3 (At4g14680); ATPS4 (At5g43780).

\section{RESULTS \\ TRANSLATION OF ATPS2 CAN BE INITIATED FROM INTERNAL START SITES}

The alignment of Arabidopsis ATPS protein sequences indicated that all four pre-proteins contained N-terminal extensions suggested to function as transit peptides for plastid targeting of polypeptides (Figure 1). Among them, only ATPS2 pre-protein contained four Met residues in its transit peptide, corresponding

\section{Transit peptide 10 \\ 20 30 \\ 40

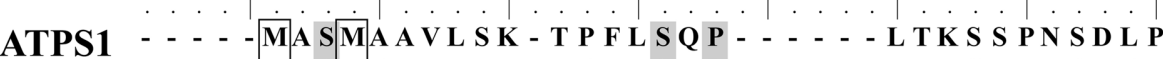 ATPS3 - - - MA SMS T V F PKP T S F I S Q P - - - - L T K S - HKS D S V ATPS4 - - . MAS S AAA I V S G S P FR S S P - . - - L I HNHHA R R Y ATPS2 MS LMI R S S Y V S H I T L F Q PRN S K P S S F T N Q I S F L S S S N N N P

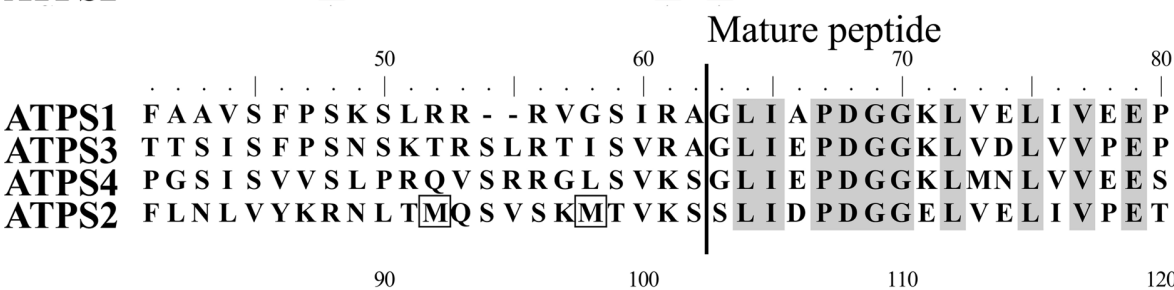 100 110 120 \\ ATPS1 KRREKKHE A AD L P - RVELT A I L QWMHVL S EGWA S P LGG ATPS3 RRR EKKHE A AD L P - R VRL T A I D L QWMH VL S E GWA S P L R G F ATPS4 RRR VMKHEAETVPAR I KLNRVDLEWVHVL S E GWA S P L KG F ATPS2 E I GVKKAES E TMP - KVKLNQ I D L EWVHVI S E GWA S P LKG F}

FIGURE 1 | Alignment of Arabidopsis ATP sulfurylase (ATPS) proteins. Arabidopsis thaliana ATPS complete protein sequences were aligned using Clustal W2. The predicted cleavage site of the transit peptide for plastid targeting is indicated. Identical residues in all four sequences are shaded and methionine residues in the transit peptides are boxed. 
to Met1, Met4, Met52, and Met58 (Figure 1). The analysis of the nucleotide sequences surrounding $\mathrm{AUG}^{\mathrm{Met}}, \mathrm{AUG}^{\mathrm{Met} 52}$, and $\mathrm{AUG}^{\mathrm{Met58}}$ codons indicated high similarities with the consensus sequence around translational initiation sites in dicot plants (Joshi et al., 1997; Hatzfeld et al., 2000). Translation of ATPS2 mRNA was therefore predicted to start at multiple sites, $\mathrm{AUG}^{\mathrm{Met} 1}$ and either $\mathrm{AUG}^{\mathrm{Met} 52}$ or $\mathrm{AUG}^{\mathrm{Met58}}$, to produce the plastidic and the cytosolic ATPS2 isoforms, respectively, in Arabidopsis.

In order to test if the translation of ATPS2 can be alternatively initiated from either $\mathrm{AUG}^{\mathrm{Met} 52}$ or $\mathrm{AUG}^{\mathrm{Met} 58}$, we constructed a

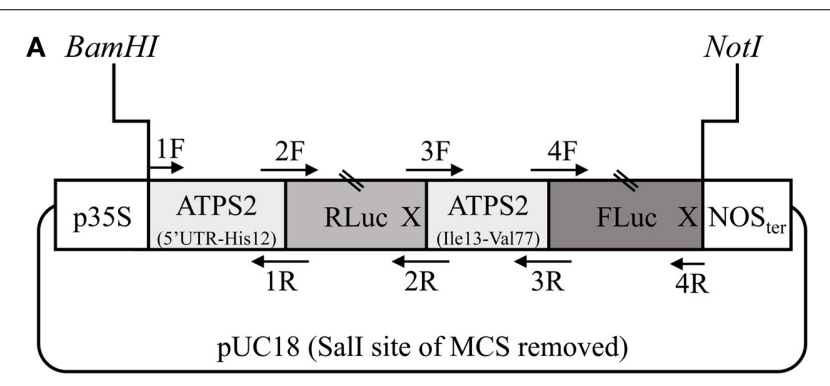

B

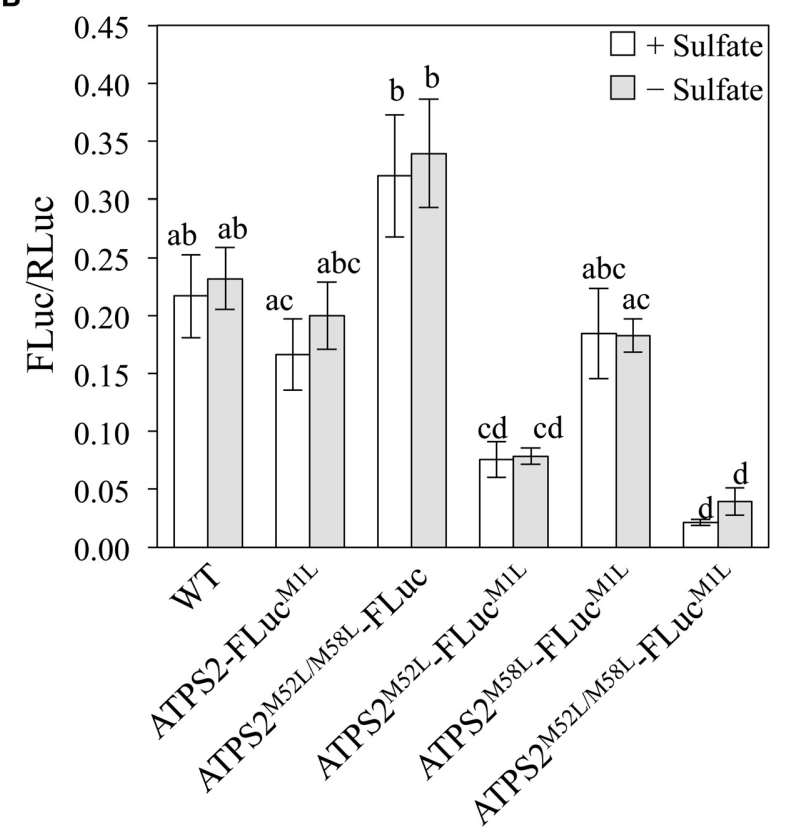

FIGURE 2 | Alternative translational initiation sites of ATPS2 determined by expression of dual-luciferase-tagged fusion constructs in Arabidopsis protoplasts. (A) Schematic representation of p35S:ATPS2-dual-Luc used for expression of tandem fusion gene ATPS2 (5'UTR-His12): Renilla luciferase:ATPS2 (lle13-Val77): firefly luciferase. Positions of annealing sites of primers (1F-4R) used for chimeric gene construction and stop codons $(X)$ are indicated. (B) FLuc activity of protein extracts from Arabidopsis protoplasts transfected with either wild-type (WT) or mutated versions of $p 35$ :ATPS2-dual-Luc chimeric genes. Results are presented as FLuc/Rluc activity ratios in the same transfection. Values indicate mean \pm SD for the results of 8 independent transfections. Values marked with different letters indicate statistically significant differences (ANOVA followed by Tukey's HSD post-hoc test; $p<0.05$ ). tandem fusion gene, $p 35 S: A T P S 2-d u a l-L u c$, that splits the portion from the $5^{\prime}$-untranslated region ( $\left.5^{\prime} \mathrm{UTR}\right)$ through the N-terminal 77-amino-acid region of ATPS2 into two parts fused separately to two luciferase reporters (Figure 2A). A 184-bp fragment of DNA, from the $5^{\prime}$ UTR to His12 of ATPS2, was cloned in frame with the coding sequence of Renilla luciferase (RLuc), and the remaining 195-bp fragment, from Ile13 to Val77 of ATPS2, was cloned in frame with the coding sequence of firefly luciferase (FLuc). This fusion construct was designed to express a tandem-fused single mRNA from the CaMV $35 \mathrm{~S}$ promoter, and to observe subsequent translation of that transcription unit into two different luciferase-fusion proteins. The fusion construct was transfected into Arabidopsis protoplasts and both luciferase activities were monitored to determine the presence of translational products, M1M4-RLuc and M52M58-FLuc. A similar bicistronic gene construct containing GFP and FLuc open reading frames has been used to characterize the ability of a viral internal ribosome entry site (IRES) to mediate cap-independent internal translational initiation in plants (Urwin et al., 2000).

The FLuc activity detected in the protoplasts indicated that M52M58-FLuc protein is produced (Figure 2B) and that an alternative translational initiation site is present and functional. To determine which of the potential start sites, $\mathrm{AUG}^{\mathrm{Met} 52}$ or $\mathrm{AUG}^{\mathrm{Met} 58}$, can be used for producing the cytosolic isoform of ATPS2 (cyt-ATPS2), missense point mutations were introduced to the AUG codons. When the AUG ${ }^{\text {Metl }}$ of FLuc was mutagenized to $\mathrm{CUG}^{\mathrm{Leu}}$ (ATPS2-FLuc ${ }^{\mathrm{M} 1 \mathrm{~L}}$ ), the FLuc/RLuc ratios were similar to or slightly lower than those in the wild type (WT). In contrast, when both $\mathrm{AUG}^{\mathrm{Met} 52}$ and $\mathrm{AUG}^{\mathrm{Met} 58}$ were mutagenized to $\mathrm{CUG}^{\mathrm{Leu}}\left(\mathrm{ATPS} 2^{\mathrm{M} 52 \mathrm{~L} / \mathrm{M} 58 \mathrm{~L}}\right.$-FLuc), the FLuc/RLuc ratios were higher than in the WT. These results suggest that the endogenous $\mathrm{AUG}^{\mathrm{Met} 1}$ of FLuc is preferentially used as a translation initiation site in this fusion construct. Therefore, $\mathrm{AUG}^{\mathrm{Met} 1}$ of FLuc was included in mutant constructs to make cross comparisons with or among the double and triple mutants. The comparisons among the three experimental groups, ATPS2-FLuc ${ }^{\mathrm{M} 1 \mathrm{~L}}$, ATPS2 ${ }^{\mathrm{M} 52 \mathrm{~L}}$ FLuc $^{\mathrm{M} 1 \mathrm{~L}}$, and ATPS $^{\mathrm{M} 58 \mathrm{~L}_{-}} \mathrm{FLuc}^{\mathrm{M} 1 \mathrm{~L}}$, indicated that the mutation of $\mathrm{AUG}^{\mathrm{Met} 52}$ had the stronger impact showing approximately a 50\% decrease in FLuc/RLuc ratios (Figure 2B). Moreover, the comparisons among ATPS2 $2^{\mathrm{M} 52 \mathrm{~L} / \mathrm{M} 58 \mathrm{~L}}$-FLuc $^{\mathrm{M} 1 \mathrm{~L}}, \mathrm{ATPS}^{\mathrm{M} 52 \mathrm{~L}}{ }_{-}$ FLuc $^{\mathrm{M} 1 \mathrm{~L}}$, and ATPS ${ }^{\mathrm{M} 58 \mathrm{~L}}-$ FLuc $^{\mathrm{M} 1 \mathrm{~L}}$ indicated that $\mathrm{AUG}^{\mathrm{Met} 52}$ was significant for translational initiation compared to $\mathrm{AUG}^{\mathrm{Met} 58}$ that only had a marginal effect (Figure 2B). These results indicated that translation of mRNA to FLuc fusion protein occurred more efficiently at $\mathrm{AUG}^{\mathrm{Met52}}$ than $\mathrm{AUG}^{\mathrm{Met} 58}$. The results obtained from this experimental system suggest that ATPS2 mRNA can be translated preferentially at AUG ${ }^{\text {Met52 }}$ to form the cytosolic ATPS2 isoform, M52-ATPS2, rather than starting at AUG ${ }^{\text {Met58 }}$ producing M58-ATPS2. In protoplasts expressing ATPS2 ${ }^{\mathrm{M} 52 \mathrm{~L} / \mathrm{M} 58 \mathrm{~L}}$-FLuc ${ }^{\mathrm{M} 1 \mathrm{~L}}$ fusion construct, FLuc activity equivalent to approximately $10 \%$ of the wild-type level was still detectable. Therefore, it cannot be ruled out that translation might have initiated at another AUG downstream of $\mathrm{AUG}^{\text {Met1 }}$ to produce a functional FLuc protein, resulting in a low residual FLuc activity. In this experiment, the protoplasts were divided into two fractions following the transfection, and incubated under sulfate-sufficient (+Sulfate) or sulfate-deficient conditions (-Sulfate). However, 
the FLuc activity was not modulated by changes in sulfate conditions.

\section{ATPS2-GFP IS DUALLY LOCALIZED IN CHLOROPLASTS AND CYTOSOL}

To determine the subcellular localizations of the alternatively translated products of ATPS2 (M1-ATPS2 and M52ATPS2 or M58-ATPS2), the 5'UTR and the N-terminal 77 -amino-acid region of ATPS2 (ATPS2 $\left(5^{\prime} \mathrm{UTR}-\mathrm{Val} 77\right)$ ) or the ATPS2 full-length (ATPS2 ${ }_{\mathrm{FL}}$ ) sequences were fused to GFP, and resultant p35S:ATPS2-GFP constructs were transiently expressed in Arabidopsis protoplasts (Figure 3). The ATPS1-GFP fusion

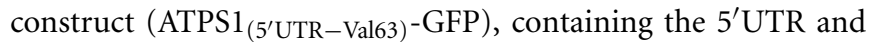
the N-terminal 63-amino-acid region of ATPS1 fused to GFP following a Val residue conserved with Val77 of ATPS2, was prepared for comparison.

In protoplasts expressing either form of wild-type ATPS2GFP fusion proteins (ATPS2 ${ }_{\left(5^{\prime} \mathrm{UTR}-\text { Val77) }\right.}$-GFP or ATPS2 $\mathrm{FL}^{-}$ GFP; Figures 3A), GFP signals were observed in both cytosol and chloroplasts ("GFP" column), where they were shown as GFP fluorescence excluded from or overlapping with chlorophyll fluorescence ("Merged" column). However, in the same transfection with the ATPS2-GFP fusion constructs, the GFP signals were found to be localized only in the cytosol in mesophyll protoplasts that contained a large number of fully developed chloroplasts (Figure 3B), unlike the dual localizations observed in protoplasts containing only a few small chloroplasts (Figure 3A). In contrast, the transfection of ATPS1 $1_{\left(5^{\prime} \mathrm{UTR}-\mathrm{Val63}\right)^{-}}$ GFP showed exclusive localization of GFP in the chloroplasts (Figure 3C).

To investigate the roles of potential start codons within the transit peptide region of ATPS2 in differentiating its localization to chloroplasts and/or cytosol, missense point mutations changing the $\mathrm{AUG}^{\mathrm{Met}}$ start codons to $\mathrm{CUG}^{\mathrm{Leu}}$ were introduced to ATPS2 $\left(5^{\prime} \mathrm{UTR}-\mathrm{V} 77\right)-\mathrm{GFP}$ and these mutant forms were expressed in protoplasts. When $\mathrm{CUG}^{\mathrm{Leu}}$ were introduced to both $\mathrm{AUG}^{\mathrm{Met1}}$ and $\mathrm{AUG}^{\mathrm{Met} 4}$ in addition to $\mathrm{AUG}^{\mathrm{Met} 1}$ of GFP (ATPS2 $^{\mathrm{M} 1 \mathrm{~L} / \mathrm{M} 4 \mathrm{~L}}-\mathrm{GFP}^{\mathrm{M} 1 \mathrm{~L}}$ ), GFP was exclusively localized in the cytosol of both cell-types with fully developed chloroplasts and small chloroplasts (Figure 3D). Furthermore, the protoplasts expressing the fusion constructs with either of these start codons intact (ATPS2 ${ }^{\mathrm{M} 1 \mathrm{~L} / \mathrm{M} 4 \mathrm{~L} / \mathrm{M} 52 \mathrm{~L}}-\mathrm{GFP}^{\mathrm{M} 1 \mathrm{~L}}$ or $\mathrm{ATPS} 2^{\mathrm{M} 1 \mathrm{~L} / \mathrm{M} 4 \mathrm{~L} / \mathrm{M} 58 \mathrm{~L}}$ $\mathrm{GFP}^{\mathrm{M} 1 \mathrm{~L}}$ ) showed GFP fluorescence localized only in the cytosol (Figure 3D). These results indicate that cytosolic ATPS2 isoform can be translated from either $\mathrm{AUG}^{\mathrm{Met} 52}$ or $\mathrm{AUG}^{\mathrm{Met} 58}$.

In contrast, the expression of a fusion protein ATPS2 $^{\mathrm{M} 52 \mathrm{~L} / \mathrm{M} 58 \mathrm{~L}}-\mathrm{GFP}^{\mathrm{M} 1 \mathrm{~L}}$ in protoplasts indicated chloroplastic localization of GFP (Figure 3E). The GFP signals were found only in protoplasts containing a few small chloroplasts, similar to the results showing dual localizations driven by the native forms of ATPS2-GFP (Figure 3A). Subcellular localizations of GFP fusion proteins were further tested using mutants, ATPS2 ${ }^{\mathrm{M} 1 \mathrm{~L} / \mathrm{M} 52 \mathrm{~L} / \mathrm{M} 58 \mathrm{~L}}$ $\mathrm{GFP}^{\mathrm{M} 1 \mathrm{~L}}$ and ATPS2 ${ }^{\mathrm{M} 4 \mathrm{~L} / \mathrm{M} 52 \mathrm{~L} / \mathrm{M} 58 \mathrm{~L}}$-GFP ${ }^{\mathrm{M} 1 \mathrm{~L}}$. When $\mathrm{AUG}^{\mathrm{Met} 4}$ was mutated, GFP was still expressed and exclusively localized in these small chloroplasts (ATPS2 ${ }^{\mathrm{M} 4 \mathrm{~L} / \mathrm{M} 52 \mathrm{~L} / \mathrm{M} 58 \mathrm{~L}}-\mathrm{GFP}^{\mathrm{M} 1 \mathrm{~L}}$; Figure 3E). However, when $\mathrm{AUG}^{\text {Met1 }}$ was mutated, no GFP fluorescence could be detected (data not shown since no GFP signals were found as in non-transfected protoplasts). This indicates that

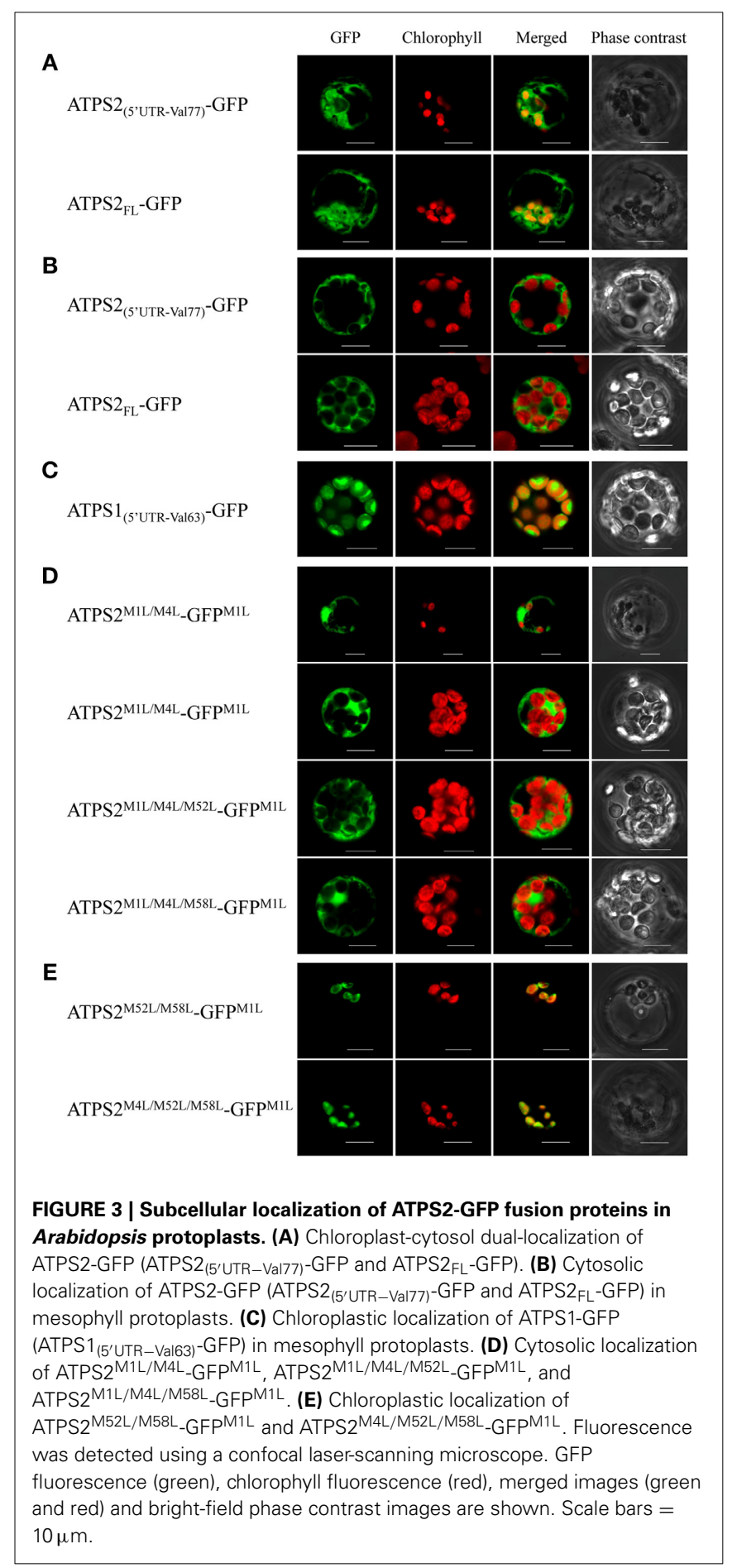

the translation of chloroplastic ATPS2 pre-protein can only be initiated from the $\mathrm{AUG}^{\mathrm{Met} 1}$ start codon.

\section{TISSUE AND SUBCELLULAR LOCALIZATIONS OF ATPS2-GFP IN PLANTS}

To further study the spatial and subcellular localization of ATPS2 in plants, ATPS2pro:ATPS2-GFP fusion constructs with or without the mutations of translational start sites were prepared (Figure S1), and stable Arabidopsis transgenic lines were obtained for the microscopic analysis (Figure 4). The 
transgenic lines expressing the ATPS1pro:ATPS1-GFP fusion construct (Kawashima et al., 2011) were used for comparison. These chimeric constructs are designed to express full-length ATPSGFP fusion proteins under control of native ATPS promoters in Arabidopsis.

The accumulations of ATPS2-GFP fusion proteins in transgenic lines were monitored by western blotting using anti-GFP antibody (Figure 4A). The fusion proteins were detected in leaves and roots of all lines except ATPS2 ${ }^{\mathrm{M} 1 \mathrm{I} / \mathrm{M} 52 \mathrm{I} / \mathrm{M} 58 \mathrm{I}}{ }_{\text {GFP, in which }}$ the fusion proteins were barely detectable in roots and only slightly produced in leaves (Figure 4A), despite the mRNAs being almost equally accumulated as those in transgenic lines made with other fusion constructs (Figure S2).

In transgenic lines expressing ATPS2-GFP fusion proteins, strong GFP fluorescence was observed in epidermal cells and guard cells as well as in vascular tissues and parenchyma cells on their abaxial side (Figure 4B). The same patterns of expression were observed in plants expressing the mutant constructs, ATPS2 ${ }^{\mathrm{M} 1 \mathrm{I} / \mathrm{M} 4 \mathrm{I}}$-GFP or ATPS2 ${ }^{\mathrm{M} 52 \mathrm{I} / \mathrm{M} 58 \mathrm{I}}-\mathrm{GFP}$, prepared to determine their cytosolic or chloroplastic localizations of GFP fusion proteins (Figure 4B). The transgenic lines expressing the ATPS1pro:ATPS1-GFP fusion construct (Kawashima et al., 2011) also showed similar tissue localization patterns of GFP (Figure 4B). In all transgenic lines, the signals of GFP were much weaker in mesophyll cells, although they were detectable.

At subcellular levels, signals of GFP were dually localized in plastids and cytosol in both leaves and roots of ATPS2-GFP lines (Figure 4C), while they were present only in plastids in ATPS1GFP lines (Figure 4D). When missense mutations $\left(\mathrm{AUC}^{\mathrm{Ile}}\right.$ ) were introduced to both $\mathrm{AUG}^{\mathrm{Met} 1}$ and $\mathrm{AUG}^{\mathrm{Met} 4}$ (ATPS2 ${ }^{\mathrm{M} 1 \mathrm{I} / \mathrm{M} 4 \mathrm{I}_{-}}$ GFP), GFP was localized exclusively in the cytosol in both leaves and roots (Figure 4E). Moreover, in transgenic lines expressing ATPS2 ${ }^{\mathrm{M} 1 \mathrm{II} / \mathrm{M} 4 \mathrm{I} / \mathrm{M} 58 \mathrm{I}}$-GFP or ATPS2 ${ }^{\mathrm{M} 1 \mathrm{I} / \mathrm{M} 4 \mathrm{I} / \mathrm{M} 52 \mathrm{I}}$-GFP fusion proteins, the GFP fluorescence was similarly detected only in the cytosol (Figure 4E). In contrast, in transgenic lines expressing the mutant construct, ATPS2 ${ }^{\mathrm{M} 52 \mathrm{I} / \mathrm{M} 58 \mathrm{I}}$-GFP, the fluorescence of GFP was localized exclusively in chloroplasts (Figure 4F). The same pattern of GFP localization was observed with ATPS2 ${ }^{\mathrm{M} 4 \mathrm{I} / \mathrm{M} 52 \mathrm{I} / \mathrm{M} 58 \mathrm{I}}$-GFP (Figure 4F). However, in ATPS2 ${ }^{\text {M1I/M52I/M58I }}$-GFP transgenic lines, GFP fluorescence could not be detected in roots, and the GFP signals in the chloroplasts in leaves were very weak (Figure $4 \mathrm{~F}$ ). The results were consistent with the low levels of ATPS2 ${ }^{\text {M1I/M52I/M58I }}$ GFP fusion protein accumulation shown in the western blots (Figure 4A). The significance of $\mathrm{AUG}^{\mathrm{Met} 1}$ for the translational initiation of chloroplast-targeted isoform was also indicated by localization of GFP signals found only in the cytosol in leaves and roots of ATPS2 ${ }^{\mathrm{MII}}$-GFP lines (Figure 4G). In contrast, the point mutation at $\mathrm{AUG}^{\mathrm{Met} 4}$ resulted in dual localization maintained as in the wild-type ATPS2-GFP, suggesting that AUG $^{\text {Met1 }}$, $\mathrm{AUG}^{\mathrm{Met} 52}$, and $\mathrm{AUG}^{\mathrm{Met58}}$ are viable as start codons (Figure 4G). The single point mutations at $\mathrm{AUG}^{\mathrm{Met} 52}$ or $\mathrm{AUG}^{\mathrm{Met} 58}$ showed similar dual localizations (Figure 4H), supporting the results obtained from triple mutants showing functional redundancies of these two start sites to produce the cytosolic isoform (Figure 4E).

\section{DISCUSSION}

The sequence analysis of four ATPS pre-proteins of Arabidopsis points to a uniqueness of ATPS2 containing four in-frame AUG codons within its transit peptide region (Met1, Met4, Met52, and Met58) (Figure 1). The present study demonstrates that translation of ATPS2 mRNA starts at multiple AUG ${ }^{\text {Met }}$ translational initiation sites and produces plastidic and cytosolic ATPS2 isoforms in Arabidopsis (Figures 2-4). This appears to happen when the nucleotide contexts surrounding the AUG start codons are favorable for initiating translations. Several studies report that alternative translations of a single mRNA can produce protein isoforms located in different subcellular compartments in plants. Pol $\gamma 2$ organellar DNA polymerases are localized in chloroplast and mitochondria following alternative translational initiation (Wamboldt et al., 2009). AtMBP-1 is alternatively translated from LOS2 transcript at the internal start codon and localizes to nucleus to modulate expression of transcriptional repressor for cold acclimation, whereas the full-length protein LOS2 functions as enolase in the glycolytic pathway in the cytosol (Lee et al., 2002; Kang et al., 2013). With regard to metabolic enzymes, NAD(P)HX dehydratase and epimerase are shown to localize in mitochondria, plastids, and cytosol by using alternative translational initiation sites (Niehaus et al., 2014).

Multiple lines of experimental evidence indicate alternative translation of ATPS2 and its relevance to plastid-cytosol dual subcellular localizations. Expression of a tandem luciferase fusion gene ATPS2 $\left(_{\left(5^{\prime} U T R-H i s 12\right): \text { Renilla }}\right.$ luciferase:ATPS2 (Ile13-Val77): firefly luciferase in Arabidopsis protoplasts suggests that M52M58-FLuc fusion protein is produced by translation initiated at internal start sites, $A_{U G}{ }^{M e t 52}$ or $\mathrm{AUG}^{\mathrm{Met} 58}$ (Figure 2). Point mutations of these alternative start sites further suggest $\mathrm{AUG}^{\mathrm{Met} 52}$ being the preferred site over $\mathrm{AUG}^{\mathrm{Met} 58}$. Furthermore, subcellular localizations of ATPS2-GFP proteins show alternative translational initiation underlying plastid-cytosol dual localizations (Figures 3, 4). The point mutation of $\mathrm{AUG}^{\mathrm{Met} 1}$ suggests the presence of this start site being essential for initiating the translation of ATPS2 pre-protein targeted to plastids. In contrast, both $\mathrm{AUG}^{\mathrm{Met} 52}$ and $\mathrm{AUG}^{\mathrm{Met} 58}$ are capable of initiating the translation of cytosolic ATPS2 isoform. These results clearly suggest bona fide relationships between the translational start sites and the duality of subcellular localizations of ATPS2 in Arabidopsis.

The exact mechanism that explains the alternative translation of ATPS2 mRNA yet remains to be verified. Leaky ribosome scanning may be one of the possible scenarios where the same ribosome reads through the entire ATPS2 mRNA to generate two ATPS2 protein isoforms with distinct subcellular localizations. Wamboldt et al. (2009) describes that such a mechanism of alternative translation of organellar DNA polymerase Poly2 mRNA allows dual localization of its translated products to mitochondria and chloroplasts in Arabidopsis. The other possible mechanism would be the presence of an internal ribosome entry site (IRES) around $\mathrm{AUG}^{\mathrm{Met} 52}$ and $\mathrm{AUG}^{\mathrm{Met} 58}$, allowing ATPS2 mRNA to produce the cytosolic ATPS2 independent of the plastid-targeted ATPS2 pre-protein. It is suggested that an IRES element in the $5^{\prime}$ UTR mediates cap-independent selective translation of a maize heat shock protein Hsp101 mRNA during heat stress (Dinkova 
A

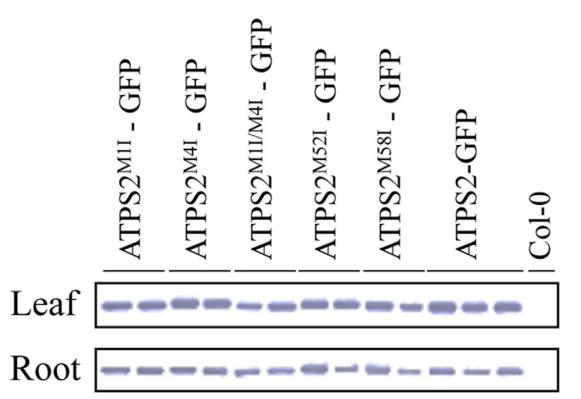

Leaf

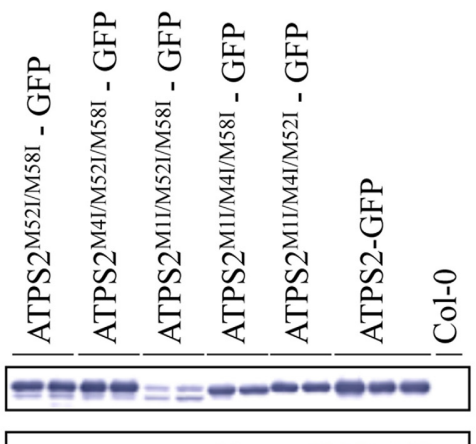

Root

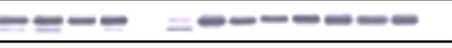

B
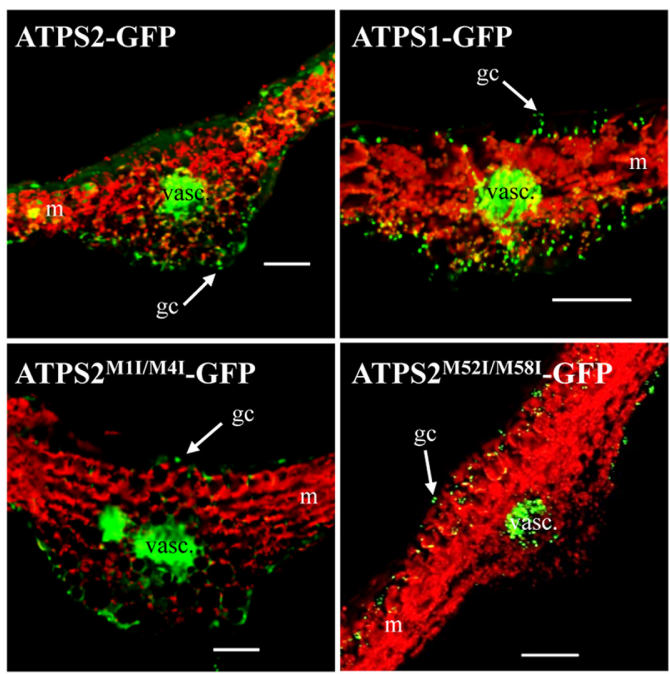

FIGURE 4 | Tissue and subcellular localization of ATPS2-GFP fusion proteins in Arabidopsis transgenic plants. (A) Detection of

ATPS2-GFP fusion proteins in each transgenic line by western blotting using anti-GFP antibody. (B) GFP fluorescence (green) observed in transverse sections of leaves from the transgenic lines expressing ATPS1pro:ATPS1:GFP, ATPS2pro:ATPS2:GFP and mutated versions (ATPS2 $^{\mathrm{M} 11 / \mathrm{M} 4 \mathrm{I}}$-GFP and ATPS2 ${ }^{\mathrm{M} 521 / \mathrm{M} 58 \mathrm{I}}$-GFP). Red indicates chlorophyll
C ATLS2-GFP

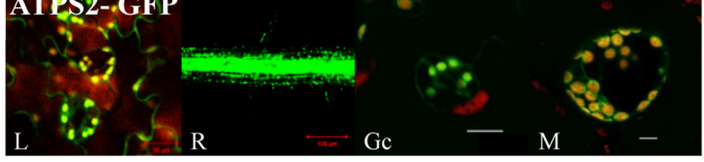

D

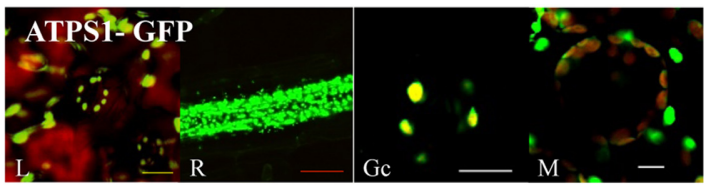

E
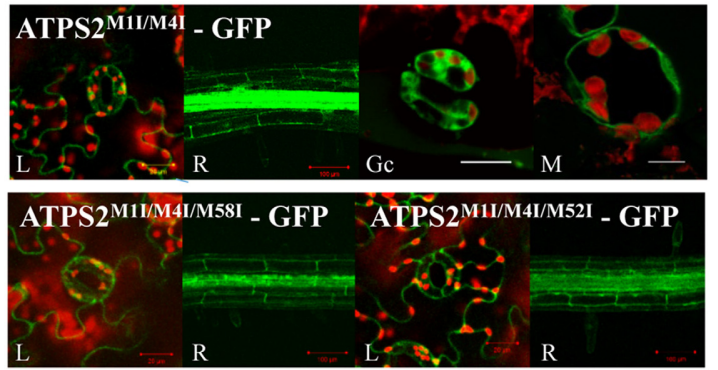

$\mathbf{F}$
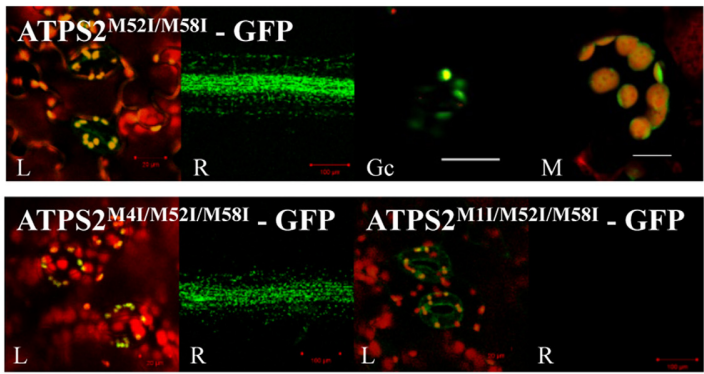

G

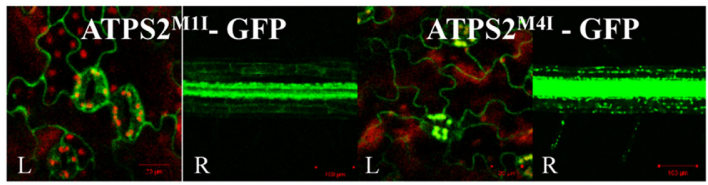

$\mathbf{H}$

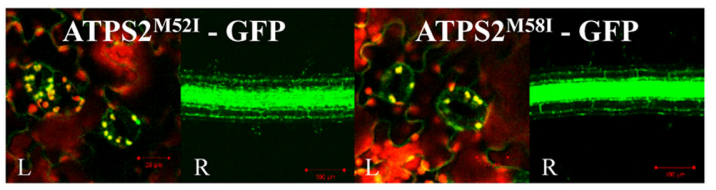

autofluorescence. Guard cells (gc), mesophyll cells (m) and vascular tissues (vasc) are indicated. Scale bars $=100 \mu \mathrm{m}$. (C-H) Subcellular localizations of GFP fluorescence (green) in leaves (L), roots (R), guard cells $(\mathrm{Gc})$, and mesophyll cells (M). Red indicates chlorophyll autofluorescence. Yellow or orange indicates overlap between green and red signals. Scales bars $=20 \mu \mathrm{m}$ (for leaves), $100 \mu \mathrm{m}$ (for roots), and $10 \mu \mathrm{m}$ (for guard cells and mesophyll cells). et al., 2005). In addition to these mechanisms, translation efficiency of a bicistronic mRNA may be affected by alteration of sequence contexts having RLuc between the first $\left(\mathrm{AUG}^{\mathrm{Met} 1}\right)$ and alternative ( $\mathrm{AUG} \mathrm{Met52}^{\mathrm{M}}$ and $\mathrm{AUG}^{\mathrm{Met} 58}$ ) start codons (Figure 2A). The overall stability of a long bicistronic mRNA with an internal stop codon can also be reduced by nonsense-mediated decay.
Molecular mechanisms of alternative translational initiations need to be investigated with precautions of considering these additional possibilities.

The transient expression of p35S:ATPS2-GFP fusion gene in Arabidopsis protoplasts indicates two patterns of subcellular localizations of GFP fluorescence depending on cell types: 
the dual localization is observed in protoplasts that contained a limited number of small chloroplasts, most likely derived from tissues that are less active in photosynthesis (Figure 3A), whereas the signal of GFP is found only in the cytosol of protoplasts containing a large number of fully developed chloroplasts, i.e., mesophyll cell protoplasts (Figure 3B). It is notable that such differential patterns of subcellular localizations are not seen with ATPS1-GFP (Figure 3C). The Arabidopsis transgenic lines expressing ATPS2pro:ATPS2-GFP fusion gene further demonstrate plastid-cytosol dual localization of ATPS2-GFP in planta. Mutations of $\mathrm{AUG}^{\mathrm{Met} 1}, \mathrm{AUG}^{\mathrm{Met52}}$, and/or $\mathrm{AUG}^{\mathrm{Met} 58}$ unequivocally indicate requirement of these potential translational start sites for producing the isoforms localized to plastids and cytosol. The analysis of transgenic lines reveal that ATPS2-GFP is dually localized with its expression being the highest in epidermal cells, guard cells, vascular tissues including bundle sheath cells, and parenchyma cells present on the abaxial side of vasculature, which are considered less active in photosynthesis (Figure 4B). This pattern of expression is partly consistent with transcriptome data indicating relatively higher expression of ATPS2 in guard cells than in mesophyll cells (Arabidopsis eFP Browser, http://bar.utoronto.ca/efp/cgi-bin/ efpWeb.cgi). These results are likely consistent with dual subcellular localizations observed in protoplasts with small chloroplasts (Figure 3A). ATPS2-GFP is still found dually localized in mesophyll cells of transgenic lines (Figure 4C), although the level of expression is lower than those observed in the cell-types or tissues described above (Figure 4B). The subcellular localization of ATPS2-GFP in mesophyll protoplasts, which appears exclusive to the cytosol (Figure 3B), must therefore be speculated to have happened under unique mechanisms. Ectopically expressed ATPS2-GFP could have been selectively translated to the cytosolic isoform or post-translationally regulated to localize the polypeptides in cytosol but not in chloroplasts under specific conditions in protoplasts. Such mechanisms that may partially differentiate subcellular localizations in different cells appear specific to ATPS2, since our results indicate that transient expression of $p 35 S-A T P S 1-G F P$ in mesophyll protoplasts demonstrates clear localization of GFP signals in chloroplasts (Figure 3C) nevertheless cell-type specificities of gene expressions are similar between ATPS1 and ATPS2 in stable transformants (Figure 4B).

As mentioned above, both ATPS1 and ATPS2 are mainly expressed in epidermal cells, guard cells, vascular tissues, and cells in their vicinity (i.e., parenchyma cells on the abaxial side of the vasculature), but to a lower extent in mesophyll cells (Figure 4B). These patterns of expression of ATPS are similar to those of APK in leaves and roots of Arabidopsis (Mugford et al., 2009). Spatial co-localization of ATPS and APK is likely in accordance with their roles in providing PAPS for secondary metabolism producing sulfated compounds. It is known that, upon herbivore attack, one of the major sulfated compounds produced in Brassicaceae are glucosinolates (GLs). GLs are hydrolyzed by myrosinase and the by-products generated upon their hydrolysis serve as defense molecules against herbivores. Although GLs are found in the entire leaf, their abundance is higher in tissues surrounding mid-veins and in the periphery of leaf (Shroff et al., 2008). The specificity of GLs distribution seems likely a mechanism to limit herbivore feeding, as they tend to feed from the edges of plant leaves. Moreover, myrosinase is localized in myrosin cells in the phloem parenchyma (Andréasson et al., 2001). The spatial separation of the myrosinase-GLs system prevents the unnecessary hydrolysis of GLs. The colocalization of ATPS and APK in the vasculature or in the vicinity of the cells expressing myrosinase is therefore indicative of its potential contribution to providing substrates for GLs biosynthesis. Furthermore, a recent study of the translatome of vascular bundle sheath cells highlights the key role of these cells in sulfur metabolism in Arabidopsis (Aubry et al., 2014).

With regard to the control of sulfate assimilation, ATPS and APK in plastids appear to be expressed in favor of synthesizing PAPS for GLs biosynthesis under sulfur-sufficient conditions. MiR395s involved in post-transcriptional gene silencing of the chloroplastic ATPS1, -3 , and -4 , are repressed under sulfursufficient conditions (Kawashima et al., 2009). Chloroplastic APK (APK1 and APK2) play significant roles in providing PAPS for GLs biosynthesis (Mugford et al., 2009) while APR is repressed under sulfur-sufficient conditions. In contrast, the physiological relevance of the presence of cytosolic ATPS and APK and their molecular regulatory mechanisms are not well-documented to date. The present study unravels the molecular identity of the cytosolic ATPS2 and proposes alternative translational initiation as an underlying mechanism for its emergence. This translational mechanism specifically allows plastid-cytosol dual localization of ATPS2, a unique non-miR395 target among the ATPS gene family members. It is noteworthy that such distinction of molecular control mechanisms is apparent among the ATPS family members nevertheless the cell-type specificity resembles each other. The results shown in this study suggest that alternative translational initiation of ATPS2 is not significantly modulated by changes in sulfur conditions. Control of chloroplastic ATPS (ATPS1, -3, and -4) thus seems important for regulation of PAPS biosynthesis in response to sulfate supply, although the potential of the miR395-mediated post-transcriptional regulation may be limited for fine-tuning the ATPS1, -3 , and -4 transcript levels (Kawashima et al., 2011). The physiological role of the cytosolic ATPS2 remains to be elucidated with relevance to its function in balancing PAPS biosynthesis between plastids and cytosol.

\section{ACKNOWLEDGMENTS}

The authors would like to thank Dr. Eva Farre (Michigan State University) for providing protocols and equipment for the bioluminescence experiments. Hideki Takahashi is supported by the National Science Foundation (MCB 1244300) and the AgBioResearch. Naoko Yoshimoto was supported by Grants-inAid for Scientific Research of Japan Society for the Promotion of Science (23770036).

\section{SUPPLEMENTARY MATERIAL}

The Supplementary Material for this article can be found online at: http://www.frontiersin.org/journal/10.3389/fpls.2014. 00750/abstract 


\section{REFERENCES}

Andréasson, E., Bolt Jørgensen, L., Höglund, A.-S., Rask, L., and Meijer, J. (2001). Different myrosinase and idioblast distribution in Arabidopsis and Brassica napus. Plant Physiol. 127, 1750-1763. doi: 10.1104/pp.010334

Aubry, S., Smith-Unna, R. D., Boursnell, C. M., Kopriva, S., and Hibberd, J. M. (2014). Transcript residency on ribosomes reveals a key role for the Arabidopsis thaliana bundle sheath in sulfur and glucosinolate metabolism. Plant J. 78, 659-673. doi: 10.1111/tpj.12502

Bradford, M. M. (1976). A rapid and sensitive method for the quantitation of microgram quantities of protein utilizing the principle of protein-dye binding. Anal. Biochem. 72, 248-254. doi: 10.1016/0003-2697(76)90527-3

Chiu, W., Niwa, Y., Zeng, W., Hirano, T., Kobayashi, H., and Sheen, J. (1996). Engineered GFP as a vital reporter in plants. Curr. Biol. 6, 325-330. doi: 10.1016/S0960-9822(02)00483-9

Clough, S. J., and Bent, A. F. (1998). Floral dip: a simplified method for Agrobacterium-mediated transformation of Arabidopsis thaliana. Plant J. 16, 735-743. doi: 10.1046/j.1365-313x.1998.00343.x

Dinkova, T. D., Zepeda, H., Martínez-Salas, E., Martínez, L. M., Nieto-Sotelo, J., and de Jiménez, E. S. (2005). Cap-independent translation of maize Hsp101. Plant J. 41, 722-731. doi: 10.1111/j.1365-313X.2005.02333.x

Gigolashvili, T., Geier, M., Ashykhmina, N., Frerigmann, H., Wulfert, S., Krueger, S., et al. (2012). The Arabidopsis thylakoid ADP/ATP carrier TAAC has an additional role in supplying plastidic phosphoadenosine 5'-phosphosulfate to the cytosol. Plant Cell 24, 4187-4204. doi: 10.1105/tpc.112.101964

Gutierrez-Marcos, J. F., Roberts, M. A, Campbell, E. I., and Wray, J. L. (1996). Three members of a novel small gene-family from Arabidopsis thaliana able to complement functionally an Escherichia coli mutant defective in PAPS reductase activity encode proteins with a thioredoxin-like domain and "APS reductase" activity. Proc. Natl. Acad. Sci. U.S.A. 93, 13377-13382. doi: 10.1073/pnas.93.23. 13377

Halkier, B. A., and Gershenzon, J. (2006). Biology and biochemistry of glucosinolates. Annu. Rev. Plant Biol. 57, 303-333. doi: 10.1146/annurev.arplant.57.032905.105228

Hatzfeld, Y., Lee, S., Lee, M., Leustek, T., and Saito, K. (2000). Functional characterization of a gene encoding a fourth ATP sulfurylase isoform from Arabidopsis thaliana. Gene 248, 51-58. doi: 10.1016/S0378-1119(00)00132-3

Höfgen, R., and Willmitzer, L. (1988). Storage of competent cells for Agrobacterium transformation. Nucleic Acids Res. 16:9877. doi: 10.1093/nar/16.20.9877

Joshi, C. P., Zhou, H., Huang, X., and Chiang, V. L. (1997). Context sequences of translation initiation codon in plants. Plant Mol. Biol. 35, 993-1001. doi: 10.1023/A:1005816823636

Kang, M., Abdelmageed, H., Lee, S., Reichert, A., Mysore, K. S., and Allen, R. D. (2013). AtMBP-1, an alternative translation product of LOS2, affects abscisic acid responses and is modulated by the E3 ubiquitin ligase AtSAP5. Plant J. 76, 481-493. doi: 10.1111/tpj.12312

Kawashima, C. G., Matthewman, C. A., Huang, S., Lee, B.-R., Yoshimoto, N., Koprivova, A., et al. (2011). Interplay of SLIM1 and miR395 in the regulation of sulfate assimilation in Arabidopsis. Plant J. 66, 863-876. doi: 10.1111/j.1365313X.2011.04547.x

Kawashima, C. G., Yoshimoto, N., Maruyama-Nakashita, A., Tsuchiya, Y. N., Saito, K., Takahashi, H., et al. (2009). Sulphur starvation induces the expression of microRNA-395 and one of its target genes but in different cell types. Plant J. 57, 313-321. doi: 10.1111/j.1365-313X.2008.03690.x

Klonus, D., Höfgen, R., Willmitzer, L., and Riesmeier, J. W. (1994). Isolation and characterization of two cDNA clones encoding ATP-sulfurylases from potato by complementation of a yeast mutant. Plant J. 6, 105-112. doi: 10.1046/j.1365313X.1994.6010105.x

Klonus, D., Riesmeier, J. W., and Willmitzer, L. (1995). A cDNA clone for an ATP-sulfurylase from Arabidopsis thaliana. Plant Physiol. 107, 653-654. doi: 10.1104/pp.107.2.653

Koncz, C., and Schell, J. (1986). The promoter of $\mathrm{T}_{L}$-DNA gene 5 controls the tissue-specific expression of chimaeric genes carried by a novel type of Agrobacterium binary vector. Mol. Genet. Genomics 204, 383-396. doi: 10.1007/BF00331014

Lee, H., Guo, Y., Ohta, M., Xiong, L., Stevenson, B., and Zhu, J.-K. (2002). LOS2, a genetic locus required for cold-responsive gene transcription encodes a bifunctional enolase. EMBO J. 21, 2692-2702. doi: 10.1093/emboj/21.11.2692

Lee, S., and Leustek, T. (1998). APS kinase from Arabidopsis thaliana: genomic organization, expression, and kinetic analysis of the recombinant enzyme. Biochem. Biophys. Res. Commun. 247, 171-175. doi: 10.1006/bbrc.199 8.8751

Leustek, T., Murillo, M., Cervantes, M., and Biology, A. M. (1994). Cloning of a cDNA encoding ATP sulfurylase from Arabidopsis thaliana by functional expression in Saccharomyces cerevisiae. Plant Physiol. 105, 897-902. doi: 10.1104/pp.105.3.897

Lillig, C. H., Schiffmann, S., Berndt, C., Berken, A., Tischka, R., and Schwenn, J. D. (2001). Molecular and catalytic properties of Arabidopsis thaliana adenylyl sulfate (APS)-kinase. Arch. Biochem. Biophys. 392, 303-310. doi: 10.1006/abbi.2001.2453

Logan, H. M., Cathala, N., Grignon, C., and Davidian, J-C. (1996). Cloning of a cDNA encoded by a member of the Arabidopsis thaliana ATP Sulfurylase multigene family: expression studies in yeast and in relation to plant sulfur nutrition. J. Biol. Chem. 271, 12227-12233. doi: 10.1074/jbc.271.21.12227

Lunn, J. E., Droux, M., Martin, J., and Douce, R. (1990). Localization of ATP sulfurylase and $O$-acetylserine(thiol)lyase in spinach leaves. Plant Physiol. 94, 1345-1352. doi: 10.1104/pp.94.3.1345

Mugford, S. G., Yoshimoto, N., Reichelt, M., Wirtz, M., Hill, L., Mugford, S. T., et al. (2009). Disruption of adenosine-5'-phosphosulfate kinase in Arabidopsis reduces levels of sulfated secondary metabolites. Plant Cell 21, 910-927. doi: 10.1105/tpc.109.065581

Murillo, M., and Leustek, T. (1995). Adenosine-5'-triphosphate-sulfurylase from Arabidopsis thaliana and Escherichia coli are functionally equivalent but structurally and kinetically divergent: nucleotide sequence of two adenosine5'-triphosphate-sulfurylase cDNAs from Arabidopsis thaliana and anaysis of a recombinant enzyme. Arch. Biochem. Biophys. 323, 195-204. doi: 10.1006/abbi.1995.0026

Niehaus, T. D., Richardson, L. G. L., Gidda, S. K., ElBadawi-Sidhu, M., Meissen, J. K., Mullen, R. T., et al. (2014). Plants utilize a highly conserved system for repair of NADH and NADPH hydrates. Plant Physiol. 165, 52-61. doi: 10.1104/pp.114.236539

Noctor, G., Mhamdi, A., Chaouch, S., Han, Y., Neukermans, J., Marquez-Garcia, B., et al. (2012). Glutathione in plants: an integrated overview. Plant Cell Environ. 35, 454-484. doi: 10.1111/j.1365-3040.2011.02400.x

Phartiyal, P., Kim, W.-S., Cahoon, R. E., Jez, J. M., and Krishnan, H. B. (2006). Soybean ATP sulfurylase, a homodimeric enzyme involved in sulfur assimilation, is abundantly expressed in roots and induced by cold treatment. Arch. Biochem. Biophys. 450, 20-29. doi: 10.1016/j.abb.2006.03.033

Renosto, F., Patel, H. C., Martin, R. L., Christopher, T., Zimmerman, G., and Segel, I. H. (1993). ATP sulfurylase from higher plants: kinetic and structural characterization of the chloroplast and cytosol enzymes from spinach leaf. Arch. Biochem. Biophys. 307, 272-285. doi: 10.1006/abbi.1993.1590

Rotte, C., and Leustek, T. (2000). Differential subcellular localization and expression of ATP sulfurylase and 5'-adenylylsulfate reductase during ontogenesis of Arabidopsis leaves indicates that cytosolic and plastid forms of ATP sulfurylase may have specialized functions. Plant Physiol. 124, 715-724. doi: 10.1104/pp.124.2.715

Setya, A., Murillo, M., and Leustek, T. (1996). Sulfate reduction in higher plants: molecular evidence for a novel 5'-adenylylsulfate reductase. Proc. Natl. Acad. Sci. U.S.A. 93, 13383-13388. doi: 10.1073/pnas.93.23.13383

Shimojima, M. (2011). Biosynthesis and functions of the plant sulfolipid. Prog. Lipid Res. 50, 234-239. doi: 10.1016/j.plipres.2011.02.003

Shroff, R., Vergara, F., Muck, A., Svatos, A., and Gershenzon, J. (2008). Nonuniform distribution of glucosinolates in Arabidopsis thaliana leaves has important consequences for plant defense. Proc. Natl. Acad. Sci. U.S.A. 105, 6196-6201. doi: 10.1073/pnas.0711730105

Suter, M., von Ballmoos, P., Kopriva, S., den Camp, R. O., Schaller, J., Kuhlemeier, C., et al. (2000). Adenosine 5'-phosphosulfate sulfotransferase and adenosine 5'-phosphosulfate reductase are identical enzymes. J. Biol. Chem. 275, 930-936. doi: $10.1074 /$ jbc. 275.2 .930

Takahashi, H., Kopriva, S., Giordano, M., Saito, K., and Hell, R. (2011). Sulfur assimilation in photosynthetic organisms: molecular functions and regulations of transporters and assimilatory enzymes. Annu. Rev. Plant Biol. 62, 157-184. doi: 10.1146/annurev-arplant-042110-103921

Urwin, P., Yi, L., Martin, H., Atkinson, H., and Gilmartin, P. M. (2000). Functional characterization of the EMCV IRES in plants. Plant J. 24, 583-589. doi: 10.1046/j.1365-313x.2000.00904.x

Valvekens, D., Van Montagu, M., and Van Lijsebettens, M. (1988). Agrobacterium tumefaciens-mediated transformation of Arabidopsis thaliana root explants by 
using kanamycin selection. Proc. Natl. Acad. Sci. U.S.A. 85, 5536-5540. doi: $10.1073 /$ pnas.85.15.5536

Wamboldt, Y., Mohammed, S., Elowsky, C., Wittgren, C., de Paula, W. B. M., and Mackenzie, S. A. (2009). Participation of leaky ribosome scanning in protein dual targeting by alternative translation initiation in higher plants. Plant Cell 21, 157-167. doi: 10.1105/tpc.108.0 63644

Yoo, S.-D., Cho, Y.-H., and Sheen, J. (2007). Arabidopsis mesophyll protoplasts: a versatile cell system for transient gene expression analysis. Nat. Protoc. 2, 1565-1572. doi: 10.1038/nprot.2007.199

Conflict of Interest Statement: The authors declare that the research was conducted in the absence of any commercial or financial relationships that could be construed as a potential conflict of interest.
Received: 29 October 2014; accepted: 08 December 2014; published online: 05 January 2015.

Citation: Bohrer A-S, Yoshimoto N, Sekiguchi A, Rykulski N, Saito K and Takahashi $H$ (2015) Alternative translational initiation of ATP sulfurylase underlying dual localization of sulfate assimilation pathways in plastids and cytosol in Arabidopsis thaliana. Front. Plant Sci. 5:750. doi: 10.3389/fpls.2014.00750

This article was submitted to Plant Physiology, a section of the journal Frontiers in Plant Science.

Copyright (c) 2015 Bohrer, Yoshimoto, Sekiguchi, Rykulski, Saito and Takahashi. This is an open-access article distributed under the terms of the Creative Commons Attribution License (CC BY). The use, distribution or reproduction in other forums is permitted, provided the original author(s) or licensor are credited and that the original publication in this journal is cited, in accordance with accepted academic practice. No use, distribution or reproduction is permitted which does not comply with these terms. 